\title{
Metacontrast with internal contours: More evidence for monotonic functions
}

\author{
LESTER A. LEFTON and JACK R. GRIFFIN \\ University of South Carolina, Columbia, South Carolina 29208
}

\begin{abstract}
Extent of metacontrast was examined as a function of introducing internal contours into the target, intercontour space between a target with contours and a mask with corresponding spokes, interstimulus intervals, and target detectability. Results showed that: (a) introducing internal contours made the targets more susceptible to metacontrast; (b) when targets are equated for detectability, there are no effects of internal contours; (c) introducing intercontour space produced less metacontrast; and (d) accuracy was a monotonic function of the delay of the mask.
\end{abstract}

Metacontrast is the phenomenal suppression of one visual stimulus by a second visual stimulus when the stimuli are nonoverlapping and fall within a critical time period. Numerous studies have shown that metacontrast is sensitive to a large number of variables, each of which affects the nature of the results obtained (Lefton, 1973). A spatial variable which has received a great amount of attention in literature is the number of internal contours in the target. Dember in a series of studies (Cox \& Dember, 1970; Ellis \& Dember, 1971; Sherrick \& Dember, 1970) has concluded: (1) the greater the number of internal contours, the more difficult the target is to detect in a nonmasking condition, and, more important (2) the greater number of internal contours in the target, the more difficult it is to mask when compared with a no-mask control condition. Thus, the targets with many contours were detected more easily than the target with few contours.

By contrast, Lefton (1974) has found opposite results. Lefton used targets that were homogeneous or contained three, six, or nine alternating segments; the mask was a black ring. His results showed that introducing internal contours in the target made the targets more susceptible to metacontrast. Thus, the target with nine contours was more easily masked than a target with three contours. These findings were in the opposite direction of those reported by Dember.

The present study is an attempt to further examine the role of internal contours in metacontrast with manipulation of intercontour space, interstimulus interval, and the detectability of the target. Subjects

The present project was supported by a grant from the National Eye Council, National Institutes of Health (1 RO1 EY 01201-01). Partial support was also provided by the National Institute of Education (NE-G-00-03-0017-01). The project presented herein was performed pursuant to a grant from NIE. Department of Health. Education. and Welfare. However, the opinions expressed herein do not necessarily reflect the position or policy of the NIE and no official endorsement by the NIE should be inferred. We thank Terry Orr for help in data collection. Requests for reprints should be sent to: Lester A. Lefton. Department of Psychology. University of South Carolina, Columbia. South Carolina 29208.

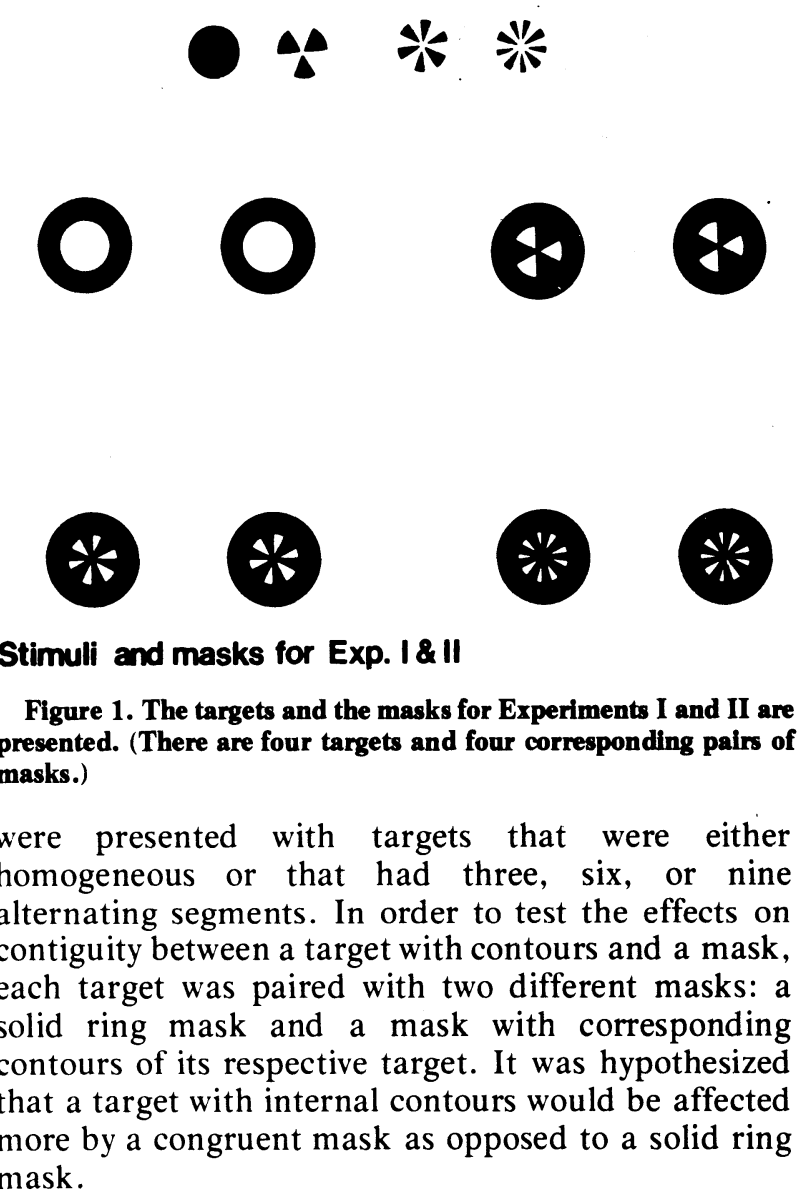

\section{GENERAL METHOD}

\section{Subjects}

Twelve undergraduates at the University of South Carolina served as subjects. All subjects were volunteers; four were.paid. All subjects had normal or corrected vision. Six subjects served in each experiment.

\section{Stimuli and Apparatus}

The stimuli were presented in a Gerbrand's four-field tachistoscope (Model T-4B). The figures that were used as masks were first drawn with black ink on white cardboard. These masks 


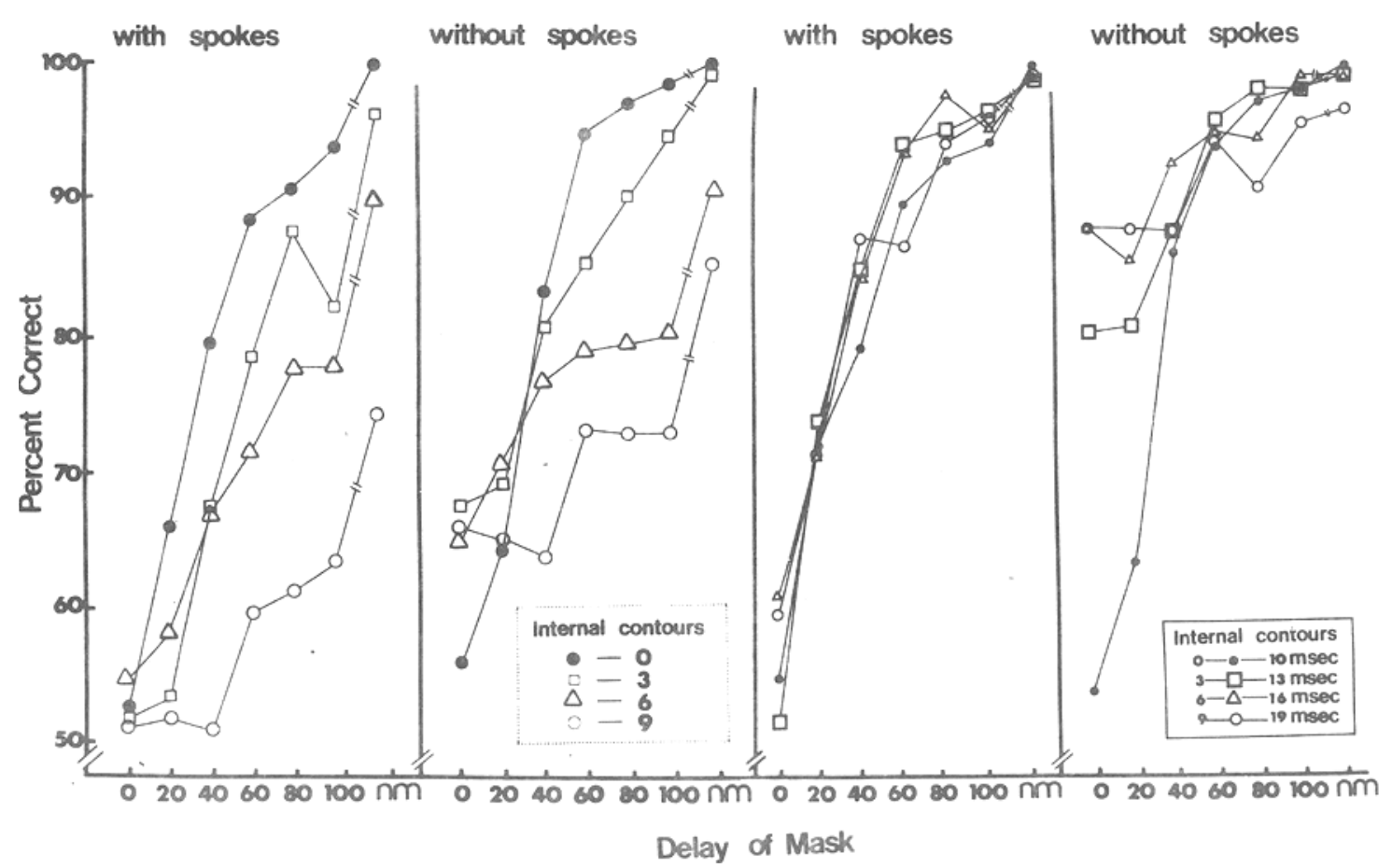

Figure 2. The mean accuracy in percent for Experiment $I$ is presented for each interstimulus interval for all four types of targets in the first and second panels. (The leftmost panel presents each target paired with its corresponding mask containing spokes. The second panel presents each target paired with the solid ring mask. The mean accuracy in percent for Experiment II is presented for each interstimulus interval for all four types of targets in the third and fourth panels. The third panel presents each target paired with its corresponding mask containing spokes. The rightmost panel presents each target paired with the solid ring mask. Each data point represents the mean of 192 observations.)

were photoprinted in order to produce uniform, black figures on a white background. Negatives of these prints were made and then rephotographed. These stimuli served as targets, thus insuring perfectly fitting nonoverlapping targets and masks. The stimuli and masks are shown in Figure 1. There were four different types of targets, homogeneous black disks or targets with three, six, or nine alternating segments. The targets with internal contours appeared similar to a windmill with alternating black and white segments. The masks were also of four different types: solid rings or rings with three, six, or nine windmill-type spokes. The targets were $1.7 \mathrm{~cm}$ in diam and at a distance of $86 \mathrm{~cm}$, subtended $1.13 \mathrm{deg}$ of visual angle.

Two masks appeared on each masking card, one to the left and one to the right of the fixation spot. The center-to-center distance of the masks was 4 deg of visual angle, and the inner diameter of the ring subtended $1.13 \mathrm{deg}$ of visual angle corresponding to the same visual angle of the targets. The width of the mask from its inner boundary to out boundary was $.47 \mathrm{deg}$.

\section{Procedure and Design}

At the beginning of each experimental session, subjects were dark adapted for $5 \mathrm{~min}$ to the ambient room illumination. During this time, the subjects were given instructions and shown the stimuli. The subjects were told on each trial to respond by indicating whether the target appeared to the left or to the right, guessing when unsure. The experimenter stressed the importance of staring at the centrally located fixation point before each trial.

Upon signal from the experimenter, subjects initiated their own trials by pressing a switch. The following sequence of stimulation would then be presented monocularly to the left eye: fixation, target, interstimulus interval, masks, and then fixation. The interstimulus interval was the light fixation field, and the background luminance of this channel was equated to that of the other channels at $.16 \mathrm{~cd} / \mathrm{m}^{2}$. The interstimulus intervals used were $0,20,40,60,80$, and $100 \mathrm{msec}$. There were never any dark intervals. A no-mask condition where only the target was presented was also included. The masks for both experiments were presented for $200 \mathrm{msec}$.

The subjects were presented with 17 blocks of trials. A block or trials consisted of a radom ordering of the six interstimulus intervals and a no-mask trial with a left and a right presentation of the target yielding 14 trials per block. The first block of trials on each experimental session was considered practice. The remaining 16 blocks of trials yielded 32 measurements for each subject at each interstimulus interval. The first two sessions of both experiments were considered practice and were not entered in the data analysis. The order of the experimental sessions was counterbalanced across subjects to reduce practice effects.

\section{EXPERIMENT I}

Each subject served for 10 experimental sessions, the first two considered practice. The remaining eight sessions were devoted to one of the possible eight combinations of number of internal contours by with-spokes or without-spokes in the mask. Thus, each target was seen twice by each subject, once with its corresponding mask and once with a mask without spokes. The target durations, for all eight conditions, were $10 \mathrm{msec}$. 


\section{Results and Discussion}

The number of errors for each experimental condition for each subject was computed and entered into an analysis of variance (number of internal contours by the presence or absence of spokes by interstimulus interval by subjects). Since there was no condition of a homogeneous target with a mask with spokes, the homogeneous target data were not included in the analysis. As is shown in Figure 2, the effect of the interstimulus interval was significant; delays of the mask increased accuracy, $F(6,30)=$ $23.46, p<.001$. Increasing the number of internal contours in the target created more metacontrast, $\mathrm{F}(2,10)=20.02, \mathrm{p}<.001$. The absence or presence of spokes in the mask also affected accuracy; the masks with spokes produced more metacontrast, $F(1,5)=36.18, p<.01$. The interaction of Interstimulus Interval by With-Spokes or Without-Spokes was also significant, $F(12,60)=4.07, p<.001$. No other interactions were significant. An analysis was done on the no-mask condition alone (internal contours by spokes by subjects). Targets with many contours were not seen as well as targets with few contours, $F(3,15)=10.57, p<.001$. The effect of spokes was also significant, $F(1,5)=18.13, p<.01$.

The results are consistent with the data reported by Lefton (1974). Increasing the number of internal contours in the target increased the ease by which targets were masked; a target with nine segments was more easily masked than a target with three segments. In addition, there was an increase in metacontrast when spokes were introduced into the mask, thus creating greater contiguity of target and mask.

When a target was presented alone, without a mask, a target with more internal contours was more difficult to see. Thus, a target with three segments was more easily detected in the no-mask condition than a target with nine segments. The results suggest that the increase in errors with an increase in internal contours may be a result of the lowered detectability of the target.

\section{EXPERIMENT II}

The purpose of this study was to repeat the conditions of Experiment I but equating the perceptibility of the targets so that differences due to internal contours might be evident without the possible confound of target perceptibility.

The same procedure and stimuli used in Experiment I were used in Experiment II. Pilot data were gathered on four subjects in order to establish a $90 \%$ detectability threshold for each target. The following values were established: homogeneous, $10 \mathrm{msec}$; three contours, $13 \mathrm{msec}$; six contours, $16 \mathrm{msec}$; and nine contours, $19 \mathrm{msec}$.

\section{Results and Discussion}

The same analysis was conducted as in
Experiment I. As is shown in Figure 2, the effects of the delay of the mask were again highly significant, $\mathrm{F}(6,30)=19.03, \mathrm{p}<.001$; there was a monotonic increase in accuracy with delays of mask. There was also an effect of the presence or absence of spokes in the mask; a mask with spokes produced more metacontrast than a mask without spokes, $F(1,5)=7.72$, $\mathrm{p}<.05$. The interaction of Interstimulus Interval by With- vs. Without-Spokes was also significant, $F(6,30)=8.91, p<.001$. Most important, the effects of the number of internal contours in the target did not reach significance $(F<1)$. An analysis was done with the no-mask condition alone which yielded no main effects of contours or spokes and nonsignificant conditions for all targets were essentially the same. Therefore, the increase in target duration to equate detectability was successful.

Lefton (1974) suggested that a target with nine alternating segments was more easily masked than a target with three segments or one that was homogeneous. Experiment II, however, shows that after equating for detectability of the target there was no effects of internal contours. By contrast with Lefton, Dember (e.g., Cox \& Dember, 1970) has suggested that an increase in the number of internal contours in a target will make the target more difficult to mask. The present experiment shows that by equating for target detectability, there was no effect of number of internal contours.

\section{GENERAL DISCUSSION}

One purpose of the present investigation was to examine the role of contiguity between the target and mask. Generally, the space between the target and mask is considered intercontour distance. The intercontour distance that is provided in the present experiment is unevenly distributed around the diameter of the target. Therefore, it is more appropriate to consider this white space as intercontour space. In both experiments, when the intercontour space was eliminated, there was an increase in metacontrast. As in manipulations of intercontour distance, decreasing the amount of intercontour space increased the extent of metacontrast. Thus, metacontrast can be enhanced through the use of a mask which eliminates intercontour space.

The results show that the number of internal contours in a target produced changes in the extent of metacontrast due to the perceptability of the target. In Experiment I, as in the study by Ellis and Dember (1971), when the target is presented alone without a mask, a target with more internal contours is more difficult to see. Thus, there is decreased detection of the target in the no-mask condition when the number of internal contours is increase. When the no-mask 
condition was equated for detectability (Experiment II), there were no significant effects of the number of internal contours.

The results show that when no-mask detectability is equated, there is no significant effect of internal contours on metacontrast, nor are there effects in any direction. These results are in contrast with those reported by Cox and Dember (1970) and by Lefton (1974). Cox and Dember found a significant effect of internal contours, Lefton found the opposite effect. Overall, the results of the present study show that introducing internal contours into a target creates changes in the detectability of the target. Once changes in detectability have been accounted for, there are no significant differences between these targets with respect to metacontrast.

A main finding of the present study was that with delays of the mask there was a monotonic increase in accuracy. Studies of metacontrast have shown two distinct types of findings. Some studies have reported U-shaped functions of accuracy (e.g., Weisstein \& Growney, 1969) and some have reported monotonic increases in accuracy as a mask is delayed (Eriksen, Becker, \& Hoffman, 1970; Lefton, 1973). The present study provides support for monotonic increases in metacontrast as a function of the delay of a mask. In both experiments, each subject showed a monotonic increase in accuracy. There was never even a hint of a U-shaped function. As has been pointed out previously (Lefton, 1974), monotonic metacontrast functions are not artifactual. They have been replicated in a number of different circumstances with many different types of stimuli, masks, and experimental questions. The critical variables which might distinguish between monotonic and U-shaped functions have yet to be determined. Schurman (1972) has suggested that the critical variable may be task differences. He has concluded that U-shaped metacontrast functions are not obtained with recognition or detection tasks. This position has been argued before (Lefton, 1973), but both U-shaped and monotonic functions have been obtained in recognition and detection tasks. One possible difference between studies which have shown U-shaped compared with monotonic metacontrast functions is the luminance levels used. In the present study, a relatively low luminance level was used to eliminate ceiling effects. Luminance levels may possibly account for a variety of differences in metacontrast studies. The possibility is under investigation.

\section{REFERENCES}

Cox, S. I., \& Dember, W. N. Backward masking of visual targets with internal contours. Psychonomic Science, 1970, 19, 255-256.

Dember, W. N., Mathews, W. D., \& Stefl, M. Backward masking and enhancement of multisegmented visual targets. Bulletin of the Psychonomic Society, 1973, 1, 45-47.

Ellis, D., \& DemBer, W. N. Backward masking of visual targets with internal contours: A replication. Psychonomic Science, 1971, 22, 91-92.

Eriksen, C. W., Becker, B. B., \& Hoffman, J. E. Safari to masking land: A hunt for the elusive U. Perception \& Psychophysics, 1970, 8, 245-250.

Lefton, L. A. Metacontrast: A review. Perception \& Psychophysics. 1973, 13, 161-171.

Lefton, L. A. Spatial factors in metacontrast. Perception \& Psychophysics, 1973, 14, 497-500.

LefToN, L. A. Internal contours, intercontour distance, and interstimulus intervals: The complex interaction in metacontrast. Journal of Experimental Psychology, 1974, 103, 891-895.

Schurman, D. L. Predictive validity of a Rashevsky-Landahl neural net: Test of a model of masking for form. Perception \& Psychophysics, 1972, 12, 183-186.

SherRick, M. F., \& DEMBER, W. N. Visual backward masking and the area-detection relation. Psychonomic Science, 1970, 19, $127-128$.

Weisstein, N., \& Growney, R. L. Apparent movement and metacontrast: A note on Kahneman's formulation. Perception \& Psychophysics, 1969, 5, 321-328.

(Received for publication September 29, 1975.) 